\title{
Note of birds collected at some places in the Territory of Papua and New Guinea
}

\author{
by Tadayuki Miyamoto \\ 要 約 ・宮 本，忠，之 \\ ニュー・ギニア島で採集した鳥類
}

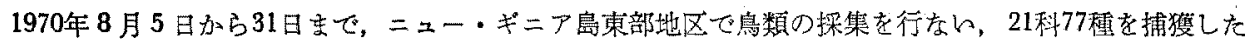
のでととに報告する。

Getting the permits from the Government of Australia, I made a small expedition for the purpose of collecting birds in New Guinea from August 5 to August 31 in 1970. So herewith I am going to inform the results of my collecting trip.

Headquarters in the Territory were,
1. Garaina :
from Aug. 7 to Aug. 9

2. A certain place near Waria River ten miles west of Garina:

\section{Ara Buka :}

from Aug. 10 to Aug. 13

4. Wau : from Aug. 14 to Aug. 19

1. Garaina: This is on the terraced hills about 2330 feet in height above sea level. The hills are surrounded by the rapid streams as if old castles were so. About eight years ago Mr. Hutton came here in order to open jungles and produce agricultural articles, and many natives worked with him. Nowadays this place has become a town in which are living twenty Gve Australians, and an airport and a school are built. And the hills are well cultivated and have huge tea plantation. In the gardens many kinds of birds occured. I was always woken by the lovely voices of birds that we could hardly hear near our dwellings in Japan. Even in midnight I was often awakened by some kinds of Owls and other animals. But it was by no means a disagreeable event for me. In this place, Kingfishers, Parrots, Cuckooshrikes, Hawks, Birds of Paradise, and Willie Wagtail, and Finches were many.

2. A certain place near Waria River ten miles west of Graina: This is the weekend place that Mr. Hutton discovered several years ago so as to enjoy his holidays with his family. There are lovely woods, grassy plains, and a river. He built a pretty hut in which were arranged the necessities of life. I spent four days in the hut. The life of this place 2500 feet high was just as that of summer resort. Various vegetables were brought in by the natives living near the village. I can't 
forget that I took a dinner under the twinkling stars hearing the murmur of Waria River and chatted away with $\mathrm{Mr}$. Abid (Who belonged to the Bishop Museum) tasting coffee. In this place, Bee-eaters, Doves, Drongos, Kingfishers, and Pied chat were many.

3. Ara Buka: This is about twenty miles far from Graina and 6000 feet in height above sea level. To get there we took cars half an hour and then walked six hours. Camping there, I expected to collect submontane specices of avifauna. In that season New Guinea is usually in rather a dry one, but this place was so much wet. Heavy rain came every night and even in daytime it often did. I was obliged to be out to collect birds in spite of getting wet. And the altitude was so high that I spent every time in wearing a sweater. In a certain village on the way to Ara Buka, we were checked in the village, and then a counselor of this village guided us for Ara Buka. He often came to our camp in Ara Buka to ask if there were else necessary in our jungle life. In this place many birds occured, for example, Honeyeaters, Flowerpeckers, Birds of Paradise, Nightjars, Parrots, Flycatchers, and Whistlers were especially many.

4. Wau: This is famous as one of resort places in the Territory, and 3600 feet in height above sea level. The weather was rather cool. There was a smart cottage like Hotel. I enjoyed a country life in this Hotel, and went into jungles in order to collect birds whenever I wished to do so. But jungles were so far from the Hotel and moreover I had no transport conveniences. So I was taken care of by the Bishop Museum which belonged to the United States of America. In Wau Flycatchers were many, especially Black Fantail, White-faced Flycatcher, and White throated Thicket-flycatcher were abundant.

According to my impression, throughout all over the Territory there lived many bush birds, but very few plain birds (both species and numbers). It might depend upon the developmental history of New Guinea. New Guninea has had few years since it was opened. So plain birds have not probably been introduced from another continents and evolved not so much.

For my trip this time and various works after that, I was helped by many per. sons and organizations that mentioned below. I am so thankful for them from the depth of my heart.

Mr. A. F. Hutton

Dr. H. Naito

Mr. H. Ohlmus

Mr. K. Kobayashi

Mr. K. Hotta

Mr. P. Shanahan 
Mr. T. Imai

Mr. P. Harvey

Mr. E. Patterson)

Department of Agriculture in the Territory of Papua \& New Guinea

Dr. Gressit

Dr. Tignar

Mr. Abid

Mrs. Yamamoto

Bishop Museum in Wau

The American Museum of Natural History

Museum of Comparative Zoology, Harvard University

In the Territory $I$ got about 200 individuals and 77 species of avifauna. So I am going to mention these birds below.

$$
\text { * Family Columbidae }
$$

1. Megaloprepia magnifica (Temmink)

Waria River: 1오, Aug. 22

오 : Wing $165 \mathrm{~mm}$, Tail $155 \mathrm{~mm}$, Culmen $17 \mathrm{~mm}$, Tarsus $18 \mathrm{~mm}$

Iris red, Bill yellow, Feet light green

2. Gallicolumba rufigula (Pucheran)

Waria River: 1우, Aug. 13

우: Wing $126 \mathrm{~mm}$, Tail $90 \mathrm{~mm}$, Culmen $18 \mathrm{~mm}$, Tarsus $27 \mathrm{~mm}$

Iris grape red, Bill dark grape, Feet dark grape

3. Gallicolumba beccarii (Salvadori)

Ara Buka: 1今, Aug. 15; 3ㅇ, Aug. 18

Wau: 1우, Aug. 22

官: Wing $105 \mathrm{~mm}$, Tail $74 \mathrm{~mm}$, Culmen $15 \mathrm{~mm}$, Tarsus $30 \mathrm{~mm}$

우: Wing 102, 105, $108 \mathrm{~mm}$, Tail 65, 72, $72 \mathrm{~mm}$, Culmen 14, 14, $14 \mathrm{~mm}$, Tarsus 28, $27,27 \mathrm{~mm}$

Iris brown, Bill black, Feet grape red

4. Gallicolumba jobiensis (A. B. Mcyer)

Garaina: 1오, Aug. 7

Waria River: 1今, Aug. 12

우: Wing $134 \mathrm{~mm}$, Tail $94 \mathrm{~mm}$, Culmen $19 \mathrm{~mm}$, Tarsus $24 \mathrm{~mm}$

令: Wing $149 \mathrm{~mm}$, Tail $99 \mathrm{~mm}$, Culmen $19 \mathrm{~mm}$, Tarsus $27 \mathrm{~mm}$

Iris brown, Bill brownish black or black, Feet reddish brown or red

5: Otidiphaps nobilis (Gould)

Ara Buka: 1今, Aug. 17

今 : Wing $189 \mathrm{~mm}$, Tail $210 \mathrm{~mm}$, Culmen $25 \mathrm{~mm}$, Tarsus $62 \mathrm{~mm}$

Iris reddish orange, Bill orange red, Feet yellow parts and grape

parts are mixed

* Family Psittacidae 
6. Trichoglossus haenatodus (Linnaeus)

Garaina: 1令, Aug. 7; 1우, Aug. 7

今: Wing $143 \mathrm{~mm}$, Tail $118 \mathrm{~mm}$, Culmen $22 \mathrm{~mm}$, Tarsus $12 \mathrm{~mm}$

우: Wing $127 \mathrm{~mm}$, Tail $101 \mathrm{~mm}$, Culmen $20 \mathrm{~mm}$, Tarsus $12 \mathrm{~mm}$

Iris red, Bill yellowish red, Feet leaden

7. Domicella hypoinochrous (Gray)

Garaina: 1令, Aug. 7

今: Wing $164 \mathrm{~mm}$, Tail $120 \mathrm{~mm}$, Culmen $30 \mathrm{~mm}$, Tarsus $22 \mathrm{~mm}$

Iris crimson red, Bill yellowish red, Feet brownish black

8. Charmosyna papou (Scopoli)

Ara Buku: 2우, Aug. 15, Aug. 16

우: Wing 125, $120 \mathrm{~mm}$, Tail 233, $226 \mathrm{~mm}$, Culmen 19, $18 \mathrm{~mm}$, Tarsus $15,12 \mathrm{~mm}$

Iris orange, Bill reddish orange, Feet orange

9. Alisterus chloropterus (Ramsay)

Garaina: 1§, Aug. 8; 1ㅇ, Aug. 8

Ara Buka: 1令, Aug. 18

今 (Garaina): Wing $198 \mathrm{~mm}$, Tail $250 \mathrm{~mm}$, Culmen $22 \mathrm{~mm}$, Tarsus $19 \mathrm{~mm}$

우 (Garaina): Wing $195 \mathrm{~mm}$, Tail $250 \mathrm{~mm}$, Culmen $24 \mathrm{~mm}$, Tarsus $19 \mathrm{~mm}$

今 (Ara Buka): Wing $185 \mathrm{~mm}$, Tail $235 \mathrm{~mm}$, Gulmen $20 \mathrm{~mm}$, Tarsus $18 \mathrm{~mm}$

Garaina's ones: Iris reddish yellow, Bill yellowish red, Feet reddish leaden

Ara Buka's one: Iris orange, Bill brownish black, Feet brownish black

* Family Cuculidae

10. Microdynamis parva (Salvadori)

Waria River: 1우, Aug. 13

우: Wing $109 \mathrm{~mm}$, Tail $120 \mathrm{~mm}$, Culmen $17 \mathrm{~mm}$, Tarsus $19 \mathrm{~mm}$

Iris brown, Bill Black, Feet yellow, Claw black

11. Eudynamis scolopacea (Linnaeus)

Wau: 1우, Aug. 24

우: Wing $178 \mathrm{~mm}$, Tail $213 \mathrm{~mm}$, Culmen $24 \mathrm{~mm}$, Tarsus $30 \mathrm{~mm}$

Iris pale, Bill brown black, Feet brownish black

$$
\text { * Family Tytonidae }
$$

12. Tyto alba (Scopoli)

Mt. Hagen: 1우, Aug. 27

오: Wing $315 \mathrm{~mm}$, Tail $126 \mathrm{~mm}$, Culmen $38 \mathrm{~mm}$, Tarsus $68 \mathrm{~mm}$

Iris brown, Bill white, Feet brown

\section{* Family Podargidae}

13. Podargus ocellatus (Quoy \& Gaimard)

Wau: 1우, Aug, 23

오: Wing $182 \mathrm{~mm}$, Tail $163 \mathrm{~mm}$, Culmen $36 \mathrm{~mm}$, Tarsus $16 \mathrm{~mm}$ 
Width of gape $45 \mathrm{~mm}$

Iris red, Bill yellow, Feet yellow

* Family Aegothelidae

14. Aegotheles albertisii (Sclater)

Ara Buka : 1今, Aug. 16

今 : Wing $174 \mathrm{~mm}$, Tail $145 \mathrm{~mm}$, Culmen $22 \mathrm{~mm}$, Tarsus $19 \mathrm{~mm}$

Iris brown, Bill brown, Feet pale brown

15. Aegotheles insignis (Salvadori)

Ara Buka: 1今, Aug. 14

令: Wing $115 \mathrm{~mm}$, Tail $106 \mathrm{~mm}$, Gulmen $17 \mathrm{~mm}$, Tarsus $20 \mathrm{~mm}$

Iris brown, Bill blackish brown, Feet yellow

\section{* Family Alcedinidae}

16. Halcyon macleayii (Jardine $\xi^{2}$ Selby)

Garaina: 1우, Aug. 9

우: Wing $93 \mathrm{~mm}$, Tail $66 \mathrm{~mm}$, Culmen $38 \mathrm{~mm}$, Tarsus $12 \mathrm{~mm}$

Iris brown, Bill black but white under the below, Feet black

17. Halcyon sancta (Vigors \& Horsfield)

Garaina: 1, Aug. 7; 2우, Aug. 7, Aug. 8

占: Wing $90 \mathrm{~mm}$, Tail $71 \mathrm{~mm}$, Culmen $38 \mathrm{~mm}$, Tarsus $11 \mathrm{~mm}$

우: Wing 91, $90 \mathrm{~mm}$, Tail $75,69 \mathrm{~mm}$, Culmen $31,35 \mathrm{~mm}$, Tarsus $11,12 \mathrm{~mm}$

Iris brown, Bill black, white at the base of the below, Feet leaden brown

18. Tanysiptera danae (Sharpe)

Waria River: 1 (immature), Aug. 12

ô: Wing $90 \mathrm{~mm}$, Tail $81 \mathrm{~mm}$ (Centre $118 \mathrm{~mm}$ ), Culmen $32 \mathrm{~mm}$, Tarsus $15 \mathrm{~mm}$

Iris brown, Bill reddish orange, Feet greenish orange

* Family Meropidae

19. Merops ornatus (Latham)

Waria River: 1今, Aug. 10;3우, Aug. 10

今: Wing $113 \mathrm{~mm}$, Tail $95 \mathrm{~mm}$ (Centre $142 \mathrm{~mm}$ ), Culmen $33 \mathrm{~mm}$, Tarsus $9 \mathrm{~mm}$

우: Wing 104, 104, $107 \mathrm{~mm}$, Tail 86, 85, $92 \mathrm{~mm}$ (Gentre 100, 95, $105 \mathrm{~mm}$ )

Culmen 33, 31, $35 \mathrm{~mm}$, Tarsus 9, 9, $9 \mathrm{~mm}$

Iris red, Bill black, Feet brownish black

* Family Campephagidae

20. Coracina boyeri (Gray)

Wau: 1우, Aug. 22

오: Wing $130 \mathrm{~mm}$, Tail $105 \mathrm{~mm}$, Culmen $19 \mathrm{~mm}$, Tarsus $20 \mathrm{~mm}$

Iris brown, Bill Black, Feet black

21. Coracina schisticeps (Gray)

Garaina: 1오, Aug. 7; 1 sex unknown (immature), Aug. 7 
우: Wing $118 \mathrm{~mm}$, Tail $107 \mathrm{~mm}$, Culmen $22 \mathrm{~mm}$, Tarsus $22 \mathrm{~mm}$

sex unknown: Wing $109 \mathrm{~mm}$, Tail $96 \mathrm{~mm}$, Culmen $20 \mathrm{~mm}$, Tarsus $21 \mathrm{~mm}$

22. Coracina caeruleogrisea (Gray)

Garaina: 1요, Aug. 8; 1우, Aug. 7

占: Wing $170 \mathrm{~mm}$, Tail $195 \mathrm{~mm}$, Culmen $40 \mathrm{~mm}$, Tarsus $28 \mathrm{~mm}$

우: Wing $170 \mathrm{~mm}$, Tail $170 \mathrm{~mm}$, Culmen $35 \mathrm{~mm}$, Tarsus $29 \mathrm{~mm}$

Iris brown, Bill black, Feet black

23. Coracina novaehollandiae (Gmelin)

Garaina: 1古, Aug. 7; 2우, Aug. 7, Aug. 9

今: Wing $199 \mathrm{~mm}$, Tail $160 \mathrm{~mm}$, Culmen $29 \mathrm{~mm}$, Tarsus $29 \mathrm{~mm}$

오: Wing 190, $200 \mathrm{~mm}$, Tail $148,150 \mathrm{~mm}$, Culmen $30,27 \mathrm{~mm}$, Tarsus $28,27 \mathrm{~mm}$

Iris brown, Bill black, Feet black

* Family Turdidae

24. Saxicola caprata (Linnaeus)

Waria River: 1今, Aug. 12

令: Wing $88 \mathrm{~mm}$, Tail $62 \mathrm{~mm}$, Culmen $18 \mathrm{~mm}$, Tarsus $23 \mathrm{~mm}$

Iris brown, Bill black, Feet black

* Family Timaliidae

25. Crateroscelis murina (Sclater)

Waria River: 1令, Aug. 12; 1우, Aug. 12

Wau : 1今, Aug. 23

占: Wing 65, $66 \mathrm{~mm}$, Tail 48, $48 \mathrm{~mm}$, Culmen 13, $14 \mathrm{~mm}$, Tarsus 27, $26 \mathrm{~mm}$

우: Wing $64 \mathrm{~mm}$, Tail $43 \mathrm{~mm}$, Culmen $14 \mathrm{~mm}$, Tarsus $24 \mathrm{~mm}$

Iris reddish brown, Bill black upper, white below, Feet pale brown

26. Crateroscelis nigronfa (Salvadori)

Ara Buka: 1今, Aug. 18

今: Wing $64 \mathrm{~mm}$, Tail $49 \mathrm{~mm}$, Gulmen $16 \mathrm{~mm}$, Tarsus $23 \mathrm{~mm}$

Iris reddish brown, Bill brownish leaden, Feet brownish black

* Family Sylviidae

27. Sericornis spilodera (Gray)

Waria River: 2우, Aug. 11

Wau: 1우, Aug. 22

우: Wing 57,61, $56 \mathrm{~mm}$, Tail 47, 49, $49 \mathrm{~mm}$, Culmen 12, 12, $12 \mathrm{~mm}$, Tarsus 18, 18, $18 \mathrm{~mm}$

Iris red, Bill pale brown, Feet light brown

28. Sericornis nouhuysi (Van Oort)

Ara Buka: 1今, Aug. 15; 1오, Aug. 16

今 : Wing $68 \mathrm{~mm}$, Tail $54 \mathrm{~mm}$, Culmen $14 \mathrm{~mm}$, Tarsus $14 \mathrm{~mm}$ 
우: Wing $65 \mathrm{~mm}$, Tail $54 \mathrm{~mm}$, Culmen $15 \mathrm{~mm}$, Tarsus $13 \mathrm{~mm}$

Iris reddish brown, Bill brownish black, Feet brown

29. Sericornis perspicillatus (Salvadori)

Ara Buka: 1요, Aug. 15; 2우, Aug. 16, Aug. 17

今 : Wing $56 \mathrm{~mm}$, Tail $47 \mathrm{~mm}$, Culmen $11 \mathrm{~mm}$, Tarsus $19 \mathrm{~mm}$

우: Wing 54, $54 \mathrm{~mm}$, Tail 48, $47 \mathrm{~mm}$, Culmen 10, $11 \mathrm{~mm}$, Tarsus 20, $19 \mathrm{~mm}$

Iris brown, Bill brownish black, Feet brown

* Family Muscicapidae

30. Rhipidura maculipectus (Gray)

Garaina: 1우, Aug. 9

우: Wing $78 \mathrm{~mm}$, Tail $97 \mathrm{~mm}$, Culmen $16 \mathrm{~mm}$, Tarsus $19 \mathrm{~mm}$

Iris brown, Bill black upper, pale brown below, Feet black

31. Rhipidura threnothorax (Muller)

Waria River: 1\$, Aug. 12

今: Wing $83 \mathrm{~mm}$, Tail $113 \mathrm{~mm}$, Gulmem $18 \mathrm{~mm}$, Tarsus $26 \mathrm{~mm}$

Iris brown, Bill black upper, pale brown below, Feet brownish black

32. Rhipidura brachyrhyncha (Schlegel)

Ara Buka: 1오, Aug. 16

Wau: 1今, Aug. 22; 1 sex unknown, Aug. 23

우: Wing $78 \mathrm{~mm}$, Tail $89 \mathrm{~mm}$, Culmen $15 \mathrm{~mm}$, Tarsus $19 \mathrm{~mm}$

占: Wing $78 \mathrm{~mm}$, Tail $100 \mathrm{~mm}$, Culmen $15 \mathrm{~mm}$, Tarsus $22 \mathrm{~mm}$

sex unknown: Wing $74 \mathrm{~mm}$, Tail $88 \mathrm{~mm}$, Culmen $14 \mathrm{~mm}$, Tarsus $21 \mathrm{~mm}$

Iris brown, Bill black, orange below, Feet brown

33. Rhipidura hyperythra (Gray)

Wau: 1우, Aug. 22

우: Wing $74 \mathrm{~mm}$, Tail $84 \mathrm{~mm}$, Culmen $14 \mathrm{~mm}$, Tarsus $15 \mathrm{~mm}$

Iris brown, Bill black upper, orange below, Feet brown

34. Rhipidura atra (Salvadori)

Wau: 1 sex unknown, Aug. 23

Ara Buka: 2今, Aug. 16, Aug. 18

sex unknown: Wing $85 \mathrm{~mm}$, Tail $89 \mathrm{~mm}$, Culmen $15 \mathrm{~mm}$, Tarsus $21 \mathrm{~mm}$

今: Wing $83,84 \mathrm{~mm}$, Tail $97,90 \mathrm{~mm}$, Culmen $14,14 \mathrm{~mm}$, Tarsus $22,20 \mathrm{~mm}$

Iris brown, Bill black upper, orange below, Feet brownish black

35. Rhipidura albolimbata (Salvadori)

Place, Date, and Sex were missed

Wing $73 \mathrm{~mm}$, Tail $82 \mathrm{~mm}$, Culmen $11 \mathrm{~mm}$, Tarsus $17 \mathrm{~mm}$

Iris brown, Bill brownish black upper, pale brown below, Feet brownish black

36. Rhipidura leucophrys (Latham)

Garaina: 1오, Aug. 8; 1 sex unknown, Aug. 8 
우: Wing $102 \mathrm{~mm}$, Tail $116 \mathrm{~mm}$, Culmen $18 \mathrm{~mm}$, Tarsus $23 \mathrm{~mm}$

sex unknown: Wing $98 \mathrm{~mm}$, Tail $100 \mathrm{~mm}$, Culmen $16 \mathrm{~mm}$, Tarsus $22 \mathrm{~mm}$

Iris brown, Bill black, Feet black

37. Monarcha axillaris (Salvadori)

Ara Buka: 1令, Aug. 15

今 : Wing $84 \mathrm{~mm}$, Tail $90 \mathrm{~mm}$, Culmen $15 \mathrm{~mm}$, Tarsus $19 \mathrm{~mm}$

Iris brown, Bill leaden but both of the tops black, Feet leaden black

38. Monarcha guttula (Garnot)

Waria River: 1今, Aug. 11

今 : Wing $82 \mathrm{~mm}$, Tail $80 \mathrm{~mm}$, Culmen $17 \mathrm{~mm}$, Tarsus $16 \mathrm{~mm}$

Iris brown, Bill leaden, Feet leaden

39. Microeca flavovirescens (Gray)

Waria River: 1今, Aug. 12

今: Wing $81 \mathrm{~mm}$, Tail $69 \mathrm{~mm}$, Gulmen $13 \mathrm{~mm}$, Tarsus $12 \mathrm{~mm}$

Iris brown, Bill black upper, orange below, Feet orange

40. Microeca papuana (Meyer)

Ara Buka: 1令, Aug. 16

今 : Wing $75 \mathrm{~mm}$, Tail $54 \mathrm{~mm}$, Culmen $13 \mathrm{~mm}$, Tarsus $15 \mathrm{~mm}$

Iris brown, Bill black, Feet orange

41. Tregellasia leucops (Salvadori)

Wau: $3 \hat{\delta}$, Aug. 22

今 : Wing 83, 86, $83 \mathrm{~mm}$, Tail 59, 54, $55 \mathrm{~mm}$, Culmen 14, 14, $14 \mathrm{~mm}$. Tarsus 22, 18, $20 \mathrm{~mm}$

Iris brown, Bill black upper, white below but black at the top of the below,

42. Peneothello cyanus (Salvadori)

Ara Buka: 3古, Aug. 15; 2우, Aug. 15, Aug. 16

令: Wing 97, 99, $101 \mathrm{~mm}$, Tail 74, 82, $79 \mathrm{~mm}$, Culmen 18, 18, $19 \mathrm{~mm}$, Tarsus 22, 27, $27 \mathrm{~mm}$

우: Wing 89, $91 \mathrm{~mm}$, Tail 79, $72 \mathrm{~mm}$, Culmen 17, $19 \mathrm{~mm}$, Tarsus 24, $24 \mathrm{~mm}$

Iris dark brown, Bill black, Feet black

43. Heteromyias albispecularis (Salvadori)

Ara Buka: 1요, Aug. 18; 1우, Aug. 17

今 : Wing $112 \mathrm{~mm}$, Tail $70 \mathrm{~mm}$, Culmen $17 \mathrm{~mm}$, Tarsus $32 \mathrm{~mm}$

우: Wing $100 \mathrm{~mm}$, Tail $67 \mathrm{~mm}$, Culmen $18 \mathrm{~mm}$, Tarsus $32 \mathrm{~mm}$

Iris brown, Bill black, or black, and white at the top of both upper and below,

Feet pale brown or pale grape

44. Pachycephalopsis poliosoma (Sharpe)

Ara Buka: 1今, Aug. 16; 2우, Aug. 15, Aug. 16

Wau: 2今, Aug. 23, Aug. 24 
今: Wing 110, 102, $105 \mathrm{~mm}$, Tail 70, 75, $73 \mathrm{~mm}$, Culmen 19, 19, $21 \mathrm{~mm}$, Tarsus 31. $31,29 \mathrm{~mm}$

우: Wing 104, $108 \mathrm{~mm}$, Tail 68, $74 \mathrm{~mm}$, Culmen $20,18 \mathrm{~mm}$, Tarsus $29,29 \mathrm{~mm}$ Iris pale yellow, Bill black, Feet leaden

* Family Pachycephalidae

45. Rhagologus leucostigma (Salvadori)

Ara Buka: 1우, Aug, 15

오 : Wing $92 \mathrm{~mm}$, tail $73 \mathrm{~mm}$, Culmen $18 \mathrm{~mm}$, Tarsus $20 \mathrm{~mm}$

Iris lemon yellow, Bill Black, Feet leaden

46. Pachycephala pectoralis (Latham)

Ara Buka: 1令, Aug. 16;1오, Aug. 16;1令 (Immature), Aug. 18

今: Wing 91, $93 \mathrm{~mm}$, Tail $70,73 \mathrm{~mm}$, Culmen $16,13 \mathrm{~mm}$, Tarsus $24,21 \mathrm{~mm}$

우: Wing $94 \mathrm{~mm}$, Tail $72 \mathrm{~mm}$, Culmen $14 \mathrm{~mm}$, Tarsus $20 \mathrm{~mm}$

Iris brown, Bill black, Feet brownish black

47. Pachycephala lorentzi (Mayr)

Ara Buka: 1今, Aug. 18

今: Wing $93 \mathrm{~mm}$, Tail $73 \mathrm{~mm}$, Culmen $13 \mathrm{~mm}$, Tarsus $21 \mathrm{~mm}$

Iris brown, Bill black, Feet leaden black

48. Pachycephala griseiceps (Gray)

Waria River: 1오, Aug. 12

우: Wing $80 \mathrm{~mm}$, Tail $69 \mathrm{~mm}$, Culmen $16 \mathrm{~mm}$, Tarsus $21 \mathrm{~mm}$

Iris reddish brown, Bill black, Feet brownish leaden

49. Myiolestes megarhynchus (Quoy \& Gaimard)

Waria River: 1\$, 1우, Aug. 12

Wau: 1오, Aug. 24

Garaina: 1송, Aug. 8

今: Wing 97, $100 \mathrm{~mm}$, Tail 86, $90 \mathrm{~mm}$, Culmen 20, $21 \mathrm{~mm}$, Tarsus 24, $24 \mathrm{~mm}$

우: Wing 93, $94 \mathrm{~mm}$, Tail 82, $85 \mathrm{~mm}$, Culmen $20,21 \mathrm{~mm}$, Tarsus $26,25 \mathrm{~mm}$

Iris red or brown, Bill leaden, Feet brownish leaden

50. Pitohui kirhocephalus (Lesson \& Garnot)

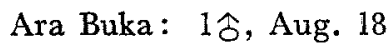

今 : Wing $132 \mathrm{~mm}$, Tail $100 \mathrm{~mm}$, Culmen $25 \mathrm{~mm}$, Tarsus $35 \mathrm{~mm}$

Iris brown, Bill leaden brown upper, pale leaden below, Feet black

51. Pitohui ferrugineus (Bonaparte)

Waria River 1今, Aug. 12

占: Wing $135 \mathrm{~mm}$, Tail $130 \mathrm{~mm}$, Culmen $30 \mathrm{~mm}$, Tarsus $32 \mathrm{~mm}$

Iris pale brown, Bill brownish black, Feet leaden

52. Pitohui nigrescens (Schlegel)

Ara Buka: 1今o, Aug. 18 
今 : Wing $142 \mathrm{~mm}$, Tail $120 \mathrm{~mm}$, Culmen $27 \mathrm{~mm}$, Tarsus $29 \mathrm{~mm}$

Iris brown, Bill black, Feet black

53. Pitohui dichrous (Bonaparte)

Waria River: 1s, Aug. 12

今 : Wing $117 \mathrm{~mm}$, Tail $113 \mathrm{~mm}$, Culmen $27 \mathrm{~mm}$, Tarsus $34 \mathrm{~mm}$

Iris grape red, Bill black, Feet black

$$
\text { * Family Grallinidae }
$$

54. Pomareopsis bruijni (Salvadori)

Ara Buka: 1§, Aug, 17; 1우, Aug. 17

今: Wing $105 \mathrm{~mm}$, Tail $91 \mathrm{~mm}$, Culmem $18 \mathrm{~mm}$, Tarsus $25 \mathrm{~mm}$

우: Wing $105 \mathrm{~mm}$, Tail $88 \mathrm{~mm}$, Gulmen $18 \mathrm{~mm}$, Tarsus $27 \mathrm{~mm}$

Iris brown, Bill leaden, Feet leaden

$$
\text { * Family Cracticidae }
$$

55. Cracticus cassicus (Boddaert)

Garaina: 1요, Aug. 9

今: Wing $117 \mathrm{~mm}$, Tail $140 \mathrm{~mm}$, Culmen $53 \mathrm{~mm}$, Tarsus $32 \mathrm{~mm}$

Iris brown, Bill pale leaden but tips black, Feet black

\section{* Family Dicruridae}

56. Chaetorhynchus papuensis (Meyer)

Waria River: 2우, Aug. 11, Aug. 12

우: Wing 108, $112 \mathrm{~mm}$, Tail 100, $104 \mathrm{~mm}$, Culmen 20, $21 \mathrm{~mm}$, Tarsus 19, $19 \mathrm{~mm}$

Iris brown, Bill black, Feet black

57. Dicrurus hottentottus (Linnaeus)

Garaina: 1우, Aug. 8

Waria River: 1우, Aug. 12

우: Wing 147, $148 \mathrm{~mm}$, Tail 138, $140 \mathrm{~mm}$, Culmen 32, $32 \mathrm{~mm}$, Tarsus $22,22 \mathrm{~mm}$

Iris red, Bill black, feet black

* Faily Ptilonorhynchidae

58. Ailuroedus buccoides (Temminck)

Wau : 1今, Aug. 22

ô: Wing $163 \mathrm{~mm}$, Tail $140 \mathrm{~mm}$, Culmen $36 \mathrm{~mm}$, Tarsus $46 \mathrm{~mm}$

Iris red, Bill pale, Feet leaden

$$
\text { * Family Meliphagidae }
$$

59. Timeliopsis fulvigula (Schlegel)

Ara Buka: 3今, Aug. 18; 1 sex unknown, Aug. 16

\&: Wing 75, 75, $82 \mathrm{~mm}$, Tail 66, 63, $79 \mathrm{~mm}$, Culmen 14, 19, $18 \mathrm{~mm}$, Tarsus 20, 23, $22 \mathrm{~mm}$

sex unknown: Wing $79 \mathrm{~mm}$, Tail $76 \mathrm{~mm}$, Culmen $17 \mathrm{~mm}$, Tarsus $20 \mathrm{~mm}$

Iris reddish orange, Bill brown upper, pale leaden below, Feet leaden brown 
60. Myzomela rosenbergii (Schlegel)

Ara Buka: 1令, Aug. 16

今 : Wing $67 \mathrm{~mm}$, Tail $38 \mathrm{~mm}$, Culmen $18 \mathrm{~mm}$, Tarsus $15 \mathrm{~mm}$

Iris brown, Bill black, Feet yellowish black

61. Toxorhamphus iliolophus (Salvadori)

Wau: 1令, Aug. 23

Waria River: 2오, Aug. 11, Aug. 12

今: Wing $72 \mathrm{~mm}$, Tail $43 \mathrm{~mm}$, Culmen $21 \mathrm{~mm}$, Tarsus $21 \mathrm{~mm}$

우: Wing $65,76 \mathrm{~mm}$, Tail $34,43 \mathrm{~mm}$, Culmen $19,21 \mathrm{~mm}$, Tarsus $18,20 \mathrm{~mm}$

Iris brown, bill black upper, same below but at the base white, Feet blackish leaden

62. Toxorhamphus poliopterus (Sharpe)

Ara Buka: 2令, Aug. 15, Aug. 16;2우, Aug 18

古: Wing 75, $71 \mathrm{~mm}$, Tail $52,48 \mathrm{~mm}$, Culmen $30,34 \mathrm{~mm}$, Tarsus $19,17 \mathrm{~mm}$

우 : Wing 67, $67 \mathrm{~mm}$, Tail 44, $39 \mathrm{~mm}$, Culmen $25,25 \mathrm{~mm}$, Tarsus $17,18 \mathrm{~mm}$

Iris brown, Bill Black, Feet leaden black

63. Melilestes megarhynchus (Gray)

Garaina: 1우, Aug. 9

Waria River: 2今, Aug. 12

오: Wing $92 \mathrm{~mm}$, Tail $89 \mathrm{~mm}$, Culmen $40 \mathrm{~mm}$, Tarsus $21 \mathrm{~mm}$

今 : Wing 98, $102 \mathrm{~mm}$, Tail 94, $88 \mathrm{~mm}$, Culmen 44, $44 \mathrm{~mm}$, Tarsus 26, $22 \mathrm{~mm}$

Iris brown or orange, Bill black, Feet leaden or leaden black

64. Melipotes fumigatus (Meyer)

Garaina: 1우, Aug. 7

Ara Buka: 1令, Aug. 17

우: Wing $110 \mathrm{~mm}$, Tail $113 \mathrm{~mm}$, Culmen $21 \mathrm{~mm}$, Tarsus $29 \mathrm{~mm}$

\$ : Wing $117 \mathrm{~mm}$, Tail $116 \mathrm{~mm}$, Culmen $21 \mathrm{~mm}$, Tarsus $29 \mathrm{~mm}$

Iris brown, Bill brownish black, Feet leaden. Yellow bare patch of skin around eye

65. Melidectes ochromelas (Meyer)

Ara Buka: 1우, Aug. 18

우: Wing $120 \mathrm{~mm}$, Tail $111 \mathrm{~mm}$, Culmen $32 \mathrm{~mm}$, Tarsus $28 \mathrm{~mm}$

Iris greenish brown, Bill pale leaden, Feet pale leaden, White bare parts are at the base of bill, and 2 red wattles hang down under the chin

66. Melidectes belfordi (De Vis)

Ara Buka: 1今, Aug. 17

今 : Wing $139 \mathrm{~mm}$, Tail $124 \mathrm{~mm}$, Culmen $39 \mathrm{~mm}$, Tarsus $33 \mathrm{~mm}$

Iris brown, Bill leaden, Feet leaden, Whitish blue bare patch of skin around eye, and 2 orange bare wattles hang down under the chin

67. Melidectes torquatus (Sclater)

Ara Buka: 1 sex unknown, Aug. 17 
Wing $119 \mathrm{~mm}$, Tail $113 \mathrm{~mm}$, Culmen $27 \mathrm{~mm}$, Tarsus $24 \mathrm{~mm}$

Iris brown, Bill pale leaden, Feet leaden, Yellow bare patch of skin around eye, and grape bare parts are at the base of bill, and 2 orange bare wattles hang down under the chin

68. Xanthotis chrysotis (Lesson \& Garnot)

Ara Buka: 1今, Aug. 17; 1우, Aug. 18

今: Wing $104 \mathrm{~mm}$, Tail $110 \mathrm{~mm}$, Gulmen $24 \mathrm{~mm}$, Tarsus $28 \mathrm{~mm}$

우: Wing $94 \mathrm{~mm}$, Tail $91 \mathrm{~mm}$, Culmen $21 \mathrm{~mm}$, Tarsus $26 \mathrm{~mm}$

Iris green, Bill black, Feet orange, Yellow bare patch of skin around eye

69. Meliphaga aruensis (Sharpe)

Garaina: 2우, Aug. 9

Waria River: 2今, Aug. 12, Aug. 13

우: Wing $85,91 \mathrm{~mm}$, Tail $78,73 \mathrm{~mm}$, Culmen $18,19 \mathrm{~mm}$, Tarsus $21,22 \mathrm{~mm}$

今: Wing 93, $95 \mathrm{~mm}$, Tail 76, $85 \mathrm{~mm}$, Culmen $17,16 \mathrm{~mm}$, Tarsus $22,21 \mathrm{~mm}$

Iris brown, Bill black, Feet brown or leaden

70. Meliphaga montana (Salvadori)

Garaina: 2今, Aug. 9; 1 sex unknown, Aug. 8

今 : Wing $85,85 \mathrm{~mm}$, Tail $77,80 \mathrm{~mm}$, Culmen $19,19 \mathrm{~mm}$, Tarsus $22,19 \mathrm{~mm}$

sex unknown: Wing $83 \mathrm{~mm}$, Tail $78 \mathrm{~mm}$, Culmen $18 \mathrm{~mm}$, Tarsus $21 \mathrm{~mm}$

Iris pale brown, Bill leaden black, Feet brown

71. Ptiloprora guisei (De Vis)

Ara Buka: 1令, Aug. 16; 1우, Aug. 17

今: Wing $88 \mathrm{~mm}$, Tail $80 \mathrm{~mm}$, Culmen $22 \mathrm{~mm}$, Tarsus $25 \mathrm{~mm}$

우: Wing $84 \mathrm{~mm}$, Tail $75 \mathrm{~mm}$, Culmen $21 \mathrm{~mm}$, Tarsus $23 \mathrm{~mm}$

Iris green, Bill black, Feet leaden

* Family Dicaeidae

72. Melanocharis longicauda (Salvadori)

Garaina: 1令, Aug. 8

Waria River: 1옹 (Immature), Aug. 12; 1우, Aug. 12

今: Wing 64, $67 \mathrm{~mm}$, Tail 59, $50 \mathrm{~mm}$, Culmen 13, $14 \mathrm{~mm}$, Tarsus $17,18 \mathrm{~mm}$

우: Wing $75 \mathrm{~mm}$, Tail $53 \mathrm{~mm}$, Culmen $13 \mathrm{~mm}$, Tarsus $17 \mathrm{~mm}$

Iris brown or orange, Bill black, Feet black or brownish black or leaden

73. Melanocharis versteri (Finsch)

Ara Buka: 2占, Aug. 16, Aug. 17; 1今 (Immature), Aug. 17; 1우, Aug. 18

今 : Wing 63, 65, $67 \mathrm{~mm}$, Tail 88, 88, $76 \mathrm{~mm}$, Culmen 14, 14, $14 \mathrm{~mm}$, Tarsus 23, 22, $23 \mathrm{~mm}$

오: Wing $74 \mathrm{~mm}$, Tail $78 \mathrm{~mm}$, Culmen $14 \mathrm{~mm}$, Tarsus $25 \mathrm{~mm}$

Iris brown, Bill black, Feet black

74. melanocharis nigra (Lesson) 
Waria River: 1우, Aug. 12

Wau: 1今, Aug. 24

今 : Wing $69 \mathrm{~mm}$, Tail $58 \mathrm{~mm}$, Culmen $18 \mathrm{~mm}$, Tarsus $14 \mathrm{~mm}$

우: Wing $64 \mathrm{~mm}$, Tail $50 \mathrm{~mm}$, Culmen $13 \mathrm{~mm}$, Tarsus $17 \mathrm{~mm}$

Iris brown, Bill black, Feet black

75. Melanocharis striativentris (Salvadori)

Ara Buka: 1令, Aug. 18; 4우, Aug. 16, Aug. 18, Aug. 18, Aug. 18,

今: Wing $76 \mathrm{~mm}$, Tail $67 \mathrm{~mm}$, Culmen $13 \mathrm{~mm}$, Tarsus $23 \mathrm{~mm}$

우: Wing 77, 72, 76, $76 \mathrm{~mm}$, Tail 62,63,67, $62 \mathrm{~mm}$, Culmen 13, 13, 15, $13 \mathrm{~mm}$

Tarsus 22, 22, 20, $21 \mathrm{~mm}$

Iris brown, Bill black, Feet leaden black

* Family Ploceidae

76. Erythrura trichroa (Kittlitz)

Waria River: 1오, Aug. 11

우: Wing $62 \mathrm{~mm}$, Tail $48 \mathrm{~mm}$, Culmen $13 \mathrm{~mm}$, Tarsus $18 \mathrm{~mm}$

Iris brown, Bill black, Feet light brown

77. Lonchura caniceps (Salvadori)

Garaina: 1우, Aug. 9; 1 sex unknown, Aug. 6

우: Wing $48 \mathrm{~mm}$, Tail $43 \mathrm{~mm}$, Culmen $12 \mathrm{~mm}$, Tarsus $13 \mathrm{~mm}$

sex unknown: Wing $45 \mathrm{~mm}$, Tail $40 \mathrm{~mm}$, Culmen $11 \mathrm{~mm}$, Tarsus $17 \mathrm{~mm}$

Iris brown, Bill black, Feet black

Bibliography :

1 Peters (J. L): Check-list of Birds of the World

2 IPC Magazines: Birds of the World

3 Rand (A. L.) \& Gilliard (E. T): Handbook of New Guinea Birds

4 Iredale $(\mathrm{T})$ : Birds of New Guinea

5 Mayr (E): Birds of Southwest Pacific

6 Hill (R): Australian Birds

7 Mayr \& Rand: Results of the Archbold Expedition 14

8 Rand : Results of the Archbold Expedition 42

9 Rand : Results of the Archbold Expedition 43

10 Rand : Results of the Archbold Expedition 44

11 Mayr \& Deusen; Results of the Archbold Expedition 74

12 Greenway $(\mathrm{J}, \mathrm{C})$ : Birds from the Coastal Range between the Markham and the Waria River, Northern New Guinea 